\title{
A GENERAL SOLUTION FOR A CLASS OF APPROXIMATION PROBLEMS
}

\author{
ANNETTE SinClaIR
}

1. Introduction. This paper generalizes a class of theorems showing the existence of an approximating function which may be required to satisfy certain auxiliary conditions.

Various theorems in analytic function theory which prove the existence of a function fulfilling specified conditions in an open set $R$ have been proved by using a method of the following type. The set $R$ is covered by an increasing sequence of sets $\left\{R_{i}\right\}$. Then the existence of a convergent sequence of functions $\left\{f_{i}(z)\right\}$ is shown such that each $f_{n}(z)$ behaves properly in $R_{n}$ and such that $\left\{f_{i}(z)\right\}$ converges to a function satisfying the required conditions everywhere in $R$. Examples of theorems in which such a method of proof can be applied are furnished by the Mittag-Leffler Theorem, the Carleman Approximation Theorem [1], some rate of growth theorems proved by P. W. Ketchum [2], and the author's generalization of Runge's Theorem [5]. W. Kaplan considered certain problems of this type and remarked, [1], that Brelot has pointed out that this type of proof is valid for approximation to a function $Q\left(x_{1}, x_{2}, \cdots, x_{n}\right)$ continuous for all $\left(x_{1}, x_{2}, \cdots, x_{n}\right)$ by a function $u\left(x_{1}, x_{2}, \cdots, x_{n+1}\right)$ harmonic for all $\left(x_{1}, x_{2}, \cdots, x_{n+1}\right)$.

The present paper attempts to give an abstract solution for this general class of problems. Examples are also given of some new results obtainable by applying Theorem 1 and fundamental approximation theorems.

In Theorem 3 approximation by an analytic function is considered on a point set $S$ consisting of an infinite number of circular discs tangent on the real axis. It is shown that a function $w(z)$ analytic at interior points of $S$ and continuous on the closure of any finite number of the circular regions-hence, continuous at their points of tangencycan be approximated by an integral function $f(z)$. Moreover, $f(z)$ can be chosen so that the approximation is stronger than uniform approximation-so that corresponding to any $\left\{\varepsilon_{i}\right\}$ there exists $f(z)$ such that

$$
|f(z)-w(z)|<\varepsilon_{i} \text { on } S_{i}
$$

where $S_{i}$ is the $i$ th. circular region.

Theorem 2 combines some previously obtained results [5] by requiring that certain auxiliary conditions be satisfied simultaneously.

Received October 25, 1956. 
Theorem 1 can sometimes also be used to show that when an approximation is known to be impossible in the infinite case that analogous results cannot hold in the finite case. An example of this usage is given.

In Part II a topological abstraction is made of Theorem 1 in which the sets of functions are topologized and the system so obtained interpreted as an inverse mapping system. It is then shown that Theorem 1 can be regarded as a special case of Theorem 5 .

\section{PART I}

2. Fundamental theorem. Let $R$ be an open subset of a topological space. A sequence of sets $\left\{R_{i}\right\}$ which satisfy the following conditions will be called an increasing sequence of $R$-covering sets.

(1) $R_{i} \subset R$;

(2) $\bar{R}_{i}$ is interior to $R_{i+1}$;

(3) $\bigcup_{i=1}^{\infty} R_{i}=R$.

$W_{1} \cup W_{2} \cup \cdots$ such that $W_{k} \cap W_{m}=\phi$ for $k \neq m$ is said to be a decomposition of a set $S$ if $S=W_{1} \cup W_{2} \cup \cdots$. An $R$-covering sequence $\left\{R_{i}\right\}$ for $R$ and a decomposition $W_{1} \cup W_{2} \cup \cdots$ of a set $S \subset R$ are said to correspond if, for every $n, W_{n} \subset R_{n}$, but $W_{n+1} \cap R_{n}=\phi$.

For a given set $S \subset R$ suppose that an increasing sequence $\left\{R_{i}\right\}$ of $R$-covering sets and a decomposition $W_{1} \cup W_{2} \cup \cdots$ of $S$ correspond. Let there be defined classes of functions $\mathscr{\mathscr { N }}_{n}$ and $\mathscr{R}_{n}$ transforming $W_{n}$ and $R_{n}$ respectively into the complex plane, $n=1,2, \ldots$. Suppose that each function of $\mathscr{R}_{n}$ defines a function of $\mathscr{R}_{n-1}, n=2,3, \cdots$.

TheOREM 1. Let $S, R, R_{n}, W_{n}, \mathscr{R}_{n}$, and $\mathscr{W}_{n}, n=1,2, \cdots$, be defined as above. Suppose that

(1) If $\left\{g_{i}(X)\right\}$ is a sequence of functions of $\mathscr{R}_{n+1}$ which converges on $R_{n+1}$ and uniformly on any closed subset of $R_{n+1}, \lim _{i \rightarrow \infty} g_{i}(X)$ defines a function of $\mathscr{R}_{n}$;

and (2) Any function defined on $R_{n}$ by an arbitrary function of the class $\mathscr{R}_{n}$ and on $W_{n+1}$ by a function of $\mathscr{W}_{n+1}$ can be uniformly approximated arbitrarily closely on $R_{n} \cup W_{n+1}$ by a function of: $\mathscr{R}_{n+1}, n=0$, $1,2, \ldots$ (where $R_{0}$ is the null set.).

Let $w(X)$ be a function defined on $S$ in such $a$ way as to determine a function of $\mathscr{W}_{i}$ for each $i$. Then, corresponding to any $\left\{\varepsilon_{i}\right\}$, there exists $r(X)$ defined on $R$ which determines a function of $\mathscr{R}_{n}$ for each $n$ such that

$$
|r(X)-w(X)|<\varepsilon_{i} \text { when } X \in W_{i}, \quad i=1,2, \cdots
$$


Proof. Suppose $\left\{\varepsilon_{i}\right\}$ and $w(X)$ preassigned. When $n$ is taken as 0 , (2) implies the existence of $r_{1}(X)$ of $\mathscr{R}_{1}$ such that

$$
\left|r_{1}(X)-w(X)\right|<\varepsilon_{1} / 2^{2} \quad \text { when } X \in W_{1} .
$$

In general, for $n=1,2, \cdots$, choose $r_{n}(X)$ of $\mathscr{R}_{n}$ so that

$$
\left|r_{n}(X)-r_{n-1}(X)\right|<\frac{\varepsilon^{(n)}}{2^{n+1}} \quad \text { on } R_{n-1}
$$

and

$$
\left|r_{n}(X)-w(X)\right|<\frac{\varepsilon^{(n)}}{2^{n+1}} \quad \text { on } W_{n},
$$

where $\varepsilon^{(n)}=\min _{\infty}\left\{\varepsilon_{1}, \varepsilon_{2}, \cdots, \varepsilon_{n}\right\}$.

Since $\left\{r_{i}(X)\right\}_{i=k+1}$ converges in $R_{k+1}$, for arbitrary $k$, and uniformly on any closed subset of $R_{k+1}$, it follows from (1) that $\lim _{i \rightarrow \infty} r_{i}(X)=r(X)$ defines a function of $\mathscr{R}_{k}$ for $k=1,2, \cdots$.

It remains to show that $r(X)$ satisfies the assigned approximation conditions. For any $k$, there exists $m>k$ so that

$$
\left|r(X)-r_{m}(X)\right|<\frac{\varepsilon^{(k)}}{2^{k}} \quad \text { when } X \in \bar{R}_{k} .
$$

Now

$$
\begin{aligned}
& |r(X)-w(X)| \leq\left|r(X)-r_{m}(X)\right|+\left|r_{m}(X)-w(X)\right| \\
\leq & \left|r(X)-r_{m}(X)\right|+\sum_{j=k+1}^{m}\left|r_{\jmath}(X)-r_{j-1}(X)\right|+\left|r_{k}(X)-w(X)\right| \\
< & \frac{\varepsilon^{(k)}}{2^{k}}+\sum_{j=k+1}^{m} \frac{\varepsilon^{j \jmath}}{2^{j+1}}+\frac{\varepsilon^{(k)}}{2^{k+1}} \quad \text { when } X \in W_{k} \subset R_{k} .
\end{aligned}
$$

Thus, $|r(X)-w(X)|<\varepsilon^{(k)} \leqq \varepsilon_{k}$ when $X \in W_{k}$. This completes the proof of the theorem.

3. Applications to specific problems. In Theorem 2 we consider approximation on a $Q$-set of the complex plane having an infinite number of components. A set $S$ is a $Q$-set if its component are closed and its set of sequential limit points lie in $C(S)$, the complement of $S$. ( $A$ sequential limit point of $S$ is a limit point of a set of points chosen one from each component of $S$. We note incidentally that a $Q$-set in the complex plane has at most a denumerable number of components and that its set of sequential limit points may separate the plane [5].)

A set in $C(S)$ is called a $B^{*}(S)$-set if it contains the set $B$ of sequential limit points of $S$ and exactly one point of each component $I_{k}(S)$ of $C(S)$ such that $I_{k}(S) \cap B=\phi$. 
The author has shown [5] that if $S$ is any $Q$-set of the complex plane and $B^{*}$ any $B^{*}(S)$-set there exists an increasing sequence $\left\{R_{i}\right\}$ of closed covering sets for $C(B)$ such that

(1) If $S_{t}$ is any component of $S$ and if $S_{t} \cap R_{n} \neq \phi$, then $S_{t} \subset R_{n}$;

(2) If $I_{r}\left(R_{n}, S\right)$ is any component of $C\left(R_{n} \cup S\right)$,

$$
I_{r}\left(R_{n}, S\right) \cap B^{*} \neq \phi .
$$

When we set $W_{i}=S \cap R_{i} \cap C\left(R_{i-1}\right)$, we obtain a decomposition $W_{1} \cup W_{2} \cup \cdots$ of $S$ which corresponds to the increasing sequence $\left\{R_{i}\right\}$ of covering sets for $C(B)$.

A function is meromorphic on a set if it is single-valued and analytic in a neighborhood of each point of the set except for poles.

Theorem 2. Suppose $S$ is a Q-set, $B$ its set of sequential limit points, and $B^{*}$ any $B^{*}(S)$-set. Let $\left\{R_{i}\right\}$ be an increasing sequence of covering sets for $C(B)$ as described above which determines the corresponding decomposition $W_{1} \cup W_{2} \cup \cdots$ of $S$. Suppose $w(z)$ is meromorphic on $S$ and that $I$ denotes the set of points of $S$ at which $w(z)$ has poles. Then, corresponding to any sequence $\left\{\varepsilon_{i}\right\}$ of positive constants, there exists $r(z)$ meromorphic in $C(B)$ and analytic in $C\left(B^{*} \cup I\right)$ such that

$$
|r(z)-w(z)|<\varepsilon_{i} \text { when } z \in\left(W_{i}-I\right), \quad i=1,2, \ldots
$$

It can be required that

(1) The poles of $r(z)$ at points of I have the same principal parts as $w(z)$;

and (2) If $K$ is an isolated interior subset of $S$ such that $K \cap I=\phi$, $r(z)$ can be chosen so that $r(k)=w(k)$ at each point $k$ of $K$. If $B^{*}$ has no limit point on $S, r(z)$ can be required to have the same multiplicities at points of $K$ as $w(z)$.

Proof. Define $\mathscr{W}_{i}$ as the set of those functions meromorphic on $W_{i}$ and analytic on $\left(W_{i}-I\right)$ which have poles with the same principal parts as $w(z)$ on $\left(I \cap W_{i}\right)$ and $k$-points with the same multiplicities as $w(z)$ on $\left(K \cap W_{i}\right)$. In $\mathscr{R}_{i}$ include those functions meromorphic on $R_{i}$ and analytic in $R_{i}-\left(I \cup B^{*}\right)$ which have poles with the same principal parts as $w(z)$ on $\left(I \cap R_{i}\right)$ and k-points with the same multiplicities as $w(z)$ on $\left(K \cap R_{i}\right)$, also those functions which are identically constant on a component of $R_{i}$ which contains no point of $I$.

Suppose $\left\{g_{i}(z)\right\}$ is a sequence of functions of $\mathscr{R}_{n+1}$ which converges in $R_{n+1}$ and uniformly on any closed subset of $R_{n+1}$ (where any points of $\left(I \cup B^{*}\right)$ are deleted from a closed subset which contains them). Then $\lim g_{i}(z)$ is meromorphic on $R_{n}$ and analytic in $R_{n}-\left(I \cup B^{*}\right)$ with poles and $k$-points identical with those of $w(z)$ at points of $I$ and $K$, except that $\lim g_{i}(z)$ may be identically constant on a component of $R_{n}$ which 
contains no points of $I$. Thus, $\lim g_{i}(z) \in \mathscr{R}_{n}$ and (1) of Theorem 1 is satisfied.

Before applying Theorem 1 it remains to show that for any $g(z)$ of $\mathscr{R}_{n}$ and $v(z)$ of $\mathscr{W}_{n+1}$, corresponding to arbitrary $\varepsilon>0$, there exists $f(z)$ of $\mathscr{R}_{n+1}$ such that

$$
\begin{gathered}
|f(z)-g(z)|<\varepsilon \text { when } z \in R_{n}, \\
\text { and }|f(z)-v(z)|<\varepsilon \text { when } z \in W_{n+1} .
\end{gathered}
$$

This follows from Walsh's generalization of Runge's Theorem [7, p. 15] and from another theorem of Walsh [7, p. 313] after it is noted that a finite number of poles on $R_{n} \cup W_{n+1}$ cause no real difficulty. Just apply the general Mittag-Leffler Theorem [4] to show the existence of a function $h(z)$ meromorphic in $C(B)$ whose poles coincide with those of $g(z)$ and $v(z)$ on $R_{n}$ and $W_{n+1}$ respectively with the same principal parts. Then define $F(z)=\left\{\begin{array}{l}g(z)-h(z) \text { on } R_{n} . \\ v(z)-h(z) \text { on } W_{n+1}\end{array}\right.$

Since $F(z)$ is analytic on $R_{n} \cup W_{n+1}$, by Walsh's generalization of Runge's Theorem [7, p. 15], there exists a rational function $k(z)$ whose poles lie in $B^{*}$ such that $|F(z)-k(z)|<\varepsilon$ when $z \in\left(R_{n} \cup W_{n+1}\right)$. Another theorem by Walsh [7, p. 313] implies that $k(z)$ can be chosen so that $k(z)=F(z)$ at points of $K$ and so that $k(z)$ has the same multiplicities at these points as $F(z)$. Set $f(z)=h(z)+k(z)$.

Now $f(z)$ is meromorphic on $R_{n+1}$ and its poles on $R_{n+1}$ lie at points of $I \cup\left(R_{n+1} \cap B^{*}\right)$ with those on $I$ having the same principal parts as $h(z)$, hence as $g(z)$ or $v(z)$, and so the same as $w(z)$. Also

$$
\begin{aligned}
|g(z)-f(z)| & =|[g(z)-h(z)]-k(z)| \\
& =|F(z)-k(z)|<\varepsilon \text { when } z \in R_{n}
\end{aligned}
$$

and similarly $|v(z)-f(z)|=|F(z)-k(z)|<\varepsilon$ when $z \in W_{n+1}$. Since $k(z)=F(z)$ at points of $K$,

$$
\begin{aligned}
f(z) & =h(z)+k(z)=h(z)+F(z)=h(z)+g(z)-h(z) \\
& =g(z) \text { on } R_{n} \cap K
\end{aligned}
$$

and, similarly, $v(z)$ on $W_{n+1} \cap K$.

This completes the proof that the hypothesis of Theorem 1 is satisfied. Hence, by Theorem 1, there is a function $r(z)$ defined on $C(B)$ (where $\infty$ is allowed as a functional value) which determines a function of $\mathscr{R}_{n}$ for each $n$ such that

$$
|r(z)-w(z)|<\varepsilon_{i} \text { when } z \in W_{i}, \quad i=1,2, \cdots .
$$

Thus, $r(z)$ is meromorphic in $C(B)$, analytic in $C\left(I \cup B^{*}\right)$, and has poles and $k$-points of $w(z)$ on $S$ as specified and also satisfies the required 
approximation condition. (In general, $r(z)$ is not identically constant on a component of $C(B)$.)

In Theorem $3 S$ consists of circular discs tangent on the real axis. More precisely, let $W_{i}=\{z /|z-i| \leq 1 / 2\}$, except that $z=i-1 / 2$ is deleted, and define $S$ as $\bigcup_{i=1}^{\infty} W_{i}$. Set $R_{i}=\{z /|z| \leq i+1 / 2\}$ and let $R$ be the finite plane. Then $\left\{R_{i}\right\}$ and the decomposition $W_{1} \cup W_{2} \cup \cdots$ of $S$ correspond.

THEOREM 3. Suppose $S$ defined as in the preceding paragraph. Let $w(z)$ be any function analytic at interior points of $S$ and continuous on the boundary except at infinity. Then, corresponding to any $\left\{\varepsilon_{i}\right\}$, there exists an integral function $r(z)$, such that $|r(z)-w(z)|<\varepsilon_{i}$ when $z \in W_{i}$ and $r(i+1 / 2)=w(i+1 / 2), i=1,2, \cdots$.

Proof. Let $\mathscr{R}_{i}$ be the set of all functions $f(z)$ analytic on $R_{i}$ such that $f(k+1 / 2)=w(k+1 / 2)$ for $k=1,2, \cdots, i$. Let $\mathscr{W}_{i}$ be the set of all functions $f(z)$ analytic at interior points of $W_{i}$ and continuous on $W_{i}$ such that $f(i+1 / 2)=w(i+1 / 2)$ and $\lim _{z \rightarrow i} f(z-1 / 2)=w(i-1 / 2)$, $(z-1 / 2) \in W_{i} i=1,2, \cdots$.

If $\left\{g_{i}(z)\right\}$, where $g_{i}(z)$ is a member of $\mathscr{R}_{n+1}$, converges on $R_{n+1}$, uniformly on any closed subset of $R_{n+1}$, lim $g_{i}(z)$ gives a function of $\mathscr{R}_{n}$,

By a theorem of Walsh [7, p. 47] a function $g(z)$ analytic interior to and continuous on a closed set $C$ which does not separate the plane and which is bounded by a finite number of Jordan curves, as is the case if $C=R_{n} \cup W_{n+1}$, can be uniformly approximated on $C$ by a polynomial $p(z)$. Then by another theorem of Walsh [7, p. 310], $p(z)$ can be chosen so that $p(k+1 / 2)=g(k+1 / 2), k=1,2, \cdots, n+1$. If $g(k+1 / 2)=w(k+1 / 2)$ then $p(z) \in \mathscr{R}_{n+1}$. Thus, the hypothesis of Theorem 1 is satisfied and the required conclusion follows.

The next theorem is an extension of the Carleman approximation theorem in that values of ahe approximating function are preassigned at certain points.

TheOREM 4. (Carleman Approximation Theorem). Let $w(x)$ be a continuous complex-valued function of $x$ for $-\infty<x<\infty$. Then, corresponding to any $\left\{\varepsilon_{i}\right\}$, there exists an integral function $f(z)$ such that

$$
|f(x)-w(x)|<\varepsilon_{i} \text { when } i-1<|x| \leq i, \quad i=1,2, \cdots,
$$

and such that $f(i)=w(i), i= \pm 1, \pm 2, \cdots$.

Proof. The proof is like that of Theorem 3 when $W_{i}$ is defined 
as $\{x|i-1<| x \mid \leq i\}$ (except that $W_{1}$ also includes the origin); $R_{i}$ as $\{z|| z \mid \leq i\} ; R$ as the finite plane; $\mathscr{W}_{i}$ as those functions continuous on $W_{i}$ such that $f( \pm i)=w( \pm i)$ and $\lim _{x \rightarrow( \pm i \mp 1)} f(x)=w( \pm i \mp 1)$; and $\mathscr{R}_{i}$ as those functions $f(z)$ analytic on $R_{i}$ such that $f(k)=w(k), k=$ $\pm 1, \pm 2, \cdots, \pm i$.

Theorem 1 can sometimes be used to show that certain requirements on the approximating function cannot, in general, be made, even when the approximation is on a set having only a finite number of components. Next an application of this type is indicated.

When approximating a function analytic and simple on each component of a closed set $C$ by a function $f(z)$ analytic in a preassigned finite region $D$ containing $C$, one cannot, in general, require that $f(z)$ be simple in $D$. To verify this we consider a $Q$-set $S$ whose components are simply connected and which has infinity as its only sequential limit point. Suppose $\left\{R_{i}\right\}$ is an increasing sequence of $R$-covering sets, as described for Theorem 2, which gives the corresponding decomposition $W_{1} \cup W_{2} \cup \cdots$ of $S$. Let $\mathscr{R}_{i}$ (and $\mathscr{W}_{i}$ ) consist of all functions analytic and simple on $R_{i}\left(W_{i}\right)$, also all constants. We note that (1) of Theorem 1 is satisfied [6, p, 203]. If (2) were also satisfied, Theorem 1 would imply that arbitrary $w(z)$ simple on $S$ could be approximated on $S$ by a function simple in the whole finite plane. Since $w(z)$ can be chosen so that $f(z)$ would necessarily have an essential singularity at $\infty$, this does not hold. We conclude that (2) is not, in general, satisfied.

Theorems 2, 3, and 4 and the illustration just stated are examples of some of the applications which can be made of Theorem 1 .

\section{PART II}

4. Topological abstraction of Theorem 1. Theorem 1 can be interpreted as a density result for a Cartesian product space. The author's original version treated the $\mathscr{R}_{i}$ 's of Theorem 1 with the respective topologies induced by the metrics

$$
d_{i}(f, g)=\sup _{x \in R_{i}}|f(X)-g(X)|
$$

as a nested sequence of spaces. The interpretation given in Theorem 5 as an inverse mapping system was suggested by Prof. Hans Samelson of the University of Michigan. In addition to having the advantage of conforming to convention, this formulation applies to classes of functions other than analytic functions.

If $\left\{W_{i}\right\}$ is any sequence of topological spaces, $W^{\infty}$ denotes the Cartesian product space $W_{1} \times W_{2} \times \cdots$. We shall be concerned with the box topology for $W^{\infty}$ in which a neighborhood of $w=\left(w_{1}, w_{2}, \cdots\right)$ is defined as $\mathrm{N}_{w_{1}}\left(W_{1}\right) \times \mathrm{N}_{w_{2}}\left(W_{2}\right) \times \cdots$. 
If $\left\{R_{i}\right\}_{i=1}^{\infty}$ is a denumerable system of $T_{2}$-spaces and if for $n=2,3, \cdots$, there is defined a continuous transformation $\prod_{n-1}^{n}$ of $R_{n}$ into $R_{n-1}$, the system $\Sigma=\left\{R_{i}, \Pi^{i}\right\}$ of the $R_{i}$ 's and $\Pi$ 's is an inverse mapping system, [3, p. 31]. The subset $\mathrm{R}$ of $R^{\infty}=R_{1} \times R_{2} \times \cdots$ of all those points $x=\left\{x_{i}\right\}$ such that $\Pi_{i}^{i+1} x_{i+1}=x_{i}$ is called the limit space of the inverse mapping system $\Sigma$.

In Theorem 5 we suppose that $R_{1}, R_{2}, \cdots$ are given sets and that for each $i$, and arbitrary points $p, q \in R_{i}$, there is defined a metric $d_{i}(p, q)$, where $\infty$ is allowed as a possible value. Then $R_{i}$ with the neighborhood system induced by $d_{i}(p, q)$ is a $T_{2}$-space. If, for $i=2,3, \cdots$, a transformation $\prod_{i-1}^{i}$ of $R_{i}$ into $R_{i-1}$ is defined which is a contraction (that is, $d_{i-1}\left(\prod_{i-1}^{i} p, \prod_{i-1}^{i} q\right) \leq d_{i}(p, q)$ ), then the $\Pi$ 's are continuous and $\left\{R_{i}, \Pi_{i-1}^{i}\right\}$ is an inverse mapping system.

Before stating Theorem 5 we note that the $R_{i}$ 's of this theorem are analogous to the $\mathscr{R}_{i}$ 's and the $W_{i}$ 's to the $\mathscr{W}_{i}$ 's of Theorem 1 .

THEOREM 5. Let $\left\{W_{i}\right\}_{i=1}^{\infty}$ be a system of topological spaces and let $\left\{R_{i}, \Pi^{i}\right\}$ be an inverse mapping system as described in the preceding paragraphs. Suppose that for each $i$ there is defined a continuous transformation $f_{i}$ which maps $R_{i}$ into $W_{i}$. Suppose also that the following conditions are satisfied:

(1) If $\left\{p_{j}^{(n)}\right\}_{j=1}^{\infty}$ is a Cauchy sequence in $R_{n}$, its image $\left\{\prod_{n=1}^{n} p_{j}^{(n)}\right\}_{j=1}^{\infty}$ is convergent in $R_{n-1}$;

(2) $f_{1}\left(R_{1}\right)$ is dense in $W_{1}$ and, when $n>1, \prod_{n-1}^{n} \times f_{n}\left(R_{n}\right)$ is dense in $R_{n-1} \times W_{n}$.

Then under the transformation $\left\{\times f_{i}\right\}$ the image of the limit space $R$ of the inverse mapping system $\Sigma$ is dense in $W^{\infty}$ by the box topology.

Proof. Let $w=\left(w_{1}, w_{2}, \cdots\right)$ be any point of $W^{\infty}$ and let $\mathrm{N}_{w}=$ $\mathrm{N}_{w_{1}}\left(W_{1}\right) \times \mathrm{N}_{w_{2}}\left(W_{2}\right) \times \cdots$ be an arbitrary neighborhood of $w$.

Since $f_{1}\left(R_{1}\right)$ is dense in $W_{1}$, there is a point $r_{1} \in R_{1}$ such that $f_{1}\left(r_{1}\right) \in \mathrm{N}_{w_{1}}\left(W_{1}\right)$. There exists $N_{r_{1}}\left(R_{1}\right) \subset N_{r_{1}}^{(1)}\left(R_{1}\right)$, where $N_{r_{1}}^{(\alpha)}=$ $\left\{p \in R_{1} / d_{1}\left(p, r_{1}\right)<\alpha\right\}$. Since $f_{1}$ is continuous and $R_{1}$ is regular, we can suppose $N_{r_{1}}\left(R_{1}\right)$ chosen so that $f_{1}\left(\bar{N}_{r_{1}}\right) \subset N_{w_{1}}\left(W_{1}\right)$.

In general, since $\prod_{n-1}^{n} \times f_{n}\left(R_{n}\right)$ is dense in $R_{n-1} \times W_{n}$, there exists $r_{n} \in R_{n}$ so that $\prod_{n-1}^{n} \times f_{n}\left(r_{n}\right) \in N_{r_{n-1}}\left(R_{n-1}\right) \times N_{w_{n}}\left(W_{n}\right)$. There exists $N_{r_{n}}\left(R_{n}\right) \subset N_{r_{n}}^{\left(1 / 2^{n-1}\right)}\left(R_{n}\right)$. Since $f_{n}$ and $\prod_{n-1}^{n}$ are continuous and $R_{n}$ is regular, we can suppose $N_{r_{n}}\left(R_{n}\right)$ chosen so that $N_{r_{n}}\left(R_{n}\right) \subset N_{r_{n}}^{\left(1 / 2 n^{n-1}\right)}\left(R_{n}\right)$ and so that

$$
\prod_{n-1}^{n} \times f_{n}\left(\bar{N}_{r_{n}}\right) \subset N_{r_{n-1}}\left(R_{n-1}\right) \times N_{w_{n}}\left(W_{n}\right) .
$$

The sequence $\left\{\prod_{n}^{n+i} r_{n+i}\right\}_{i=0}^{\infty}$, where $\prod_{n}^{n+i}=\prod_{n}^{n+1} \cdots \prod_{n+i-1}^{n+i}$, is a Cauchy 
sequence in $R_{n}$. For, corresponding to any $\varepsilon>0$, there exists $m>n$ so that $1 /\left(2^{m-2}\right)<\varepsilon ;$ then

$$
\begin{aligned}
& d_{n}\left(\prod_{n}^{m+k} r_{m+k}, \prod_{n}^{m} r_{m}\right) \leq \sum_{i=0}^{k-1} d_{n}\left(\prod_{n}^{m+i+1} r_{m+i+1}, \prod_{n}^{m+i} r_{m+i}\right) \\
\leq & \sum_{i=0}^{k-1} d_{m+i}\left(\prod_{m+i}^{m+i+1} r_{m+i+1}, r_{m+i}\right)<\sum_{i=0}^{k-1} \frac{1}{2^{m+i-1}}<\frac{1}{2^{m-2}}<\varepsilon .
\end{aligned}
$$

The first inequality follows from the triangle inequality, the second from the fact that the II's are contractions, and the third holds since $\prod_{m+i}^{m+i+1} r_{m+i+1} \in N_{r_{m+i}}\left(R_{m+1}\right) \subset N_{r_{m+i}^{((1 / 2) m+i-1)}}$.

By (1) the image of the Cauchy sequence above is convergent in $R_{n-1}$. Hence, we let $r^{(n-1)}$ denote $\lim _{i \rightarrow \infty} \prod_{n-1}^{n+i} r_{n+i}$ in $R_{n-1}$. Now $r^{(n-1)} \in \bar{N}_{r_{n-1}}\left(R_{n-1}\right)$ and $f_{n-1}\left(\bar{N}_{r_{n-1}}\left(R_{n-1}\right)\right) \subset N_{w_{n-1}}\left(W_{n-1}\right)$. Hence, $f_{n-1} r^{(n-1)} \in N_{w_{n-1}}\left(W_{n-1}\right)$.

To complete the proof of the theorem it is sufficient to show that $\left\{r^{(i-1)}\right\}_{i=2}^{\infty}$ belongs to the limit space, that is, that $r^{(n-2)}=\Pi_{n-2}^{n-1} \gamma^{(n-1)}$ for $n=3,4, \cdots$. Since $\left\{\prod_{n-1}^{n+i} r_{n+i}\right\}$ converges to $r^{(n-1)}$ in $R_{n-i}$, corresponding to any $\delta>0$, there exists $k$ such that $i>k$ implies $d_{n-1}\left(\prod_{n-1}^{n+i} r_{n+1}, r^{(n-1)}\right)<\delta$. Then, since the $\pi$ 's are contractions, $d_{n-2}\left(\prod_{n-2}^{n+i} r_{n+i}, \prod_{n-2}^{n-1} r^{(n-1)}\right)<\delta$ for all $i>k$. Now $\lim \prod_{n-2}^{n+i} r_{n+i}$ is unique in $R_{n-2}$, and so $\prod_{n-2}^{n-1} r^{(n-1)}=$ $\lim _{i \rightarrow \infty} \prod_{n-2}^{n+i} r_{n+i}=r^{(n-2)}$. This completes the proof of Theorem 5 .

If a function of $\mathscr{R}_{n}$ defines a function of $\mathscr{H}_{n}, n=1,2, \cdots$, Theorem 1 can be obtained from Theorem 5. Since each function of $\mathscr{R}_{n}$ in Theorem 1 defines a function of $\mathscr{R}_{n-1}$ (and in the case just specified, also $\mathscr{W}_{n}$ ), transformations $\prod_{n-1}^{n}$ and $f_{n}$ are determined of $\mathscr{R}_{n}$ into $\mathscr{R}_{n-1}$ and $\mathscr{W}_{n}$. Let us define a metric $d_{n}(f, g)$ for each $\mathscr{R}_{n}$ (also $\mathscr{W}_{n}$ ) as $\sup _{X \in R_{n}\left(\text { or } w_{n}\right)}|f(X)-g(X)|$. Thus, $\mathrm{T}_{2}$-topologies are determined for $\mathscr{R}_{n}$ and $\mathscr{W}_{n}$ respectively. If $f, g \in \mathscr{R}_{n}$ then,

$$
\sup _{X \in R_{n-j} \text { or } W_{n}}|f(X)-g(X)| \leq \sup _{X \in R_{n}}|f(X)-g(X)|
$$

and so $\prod_{n-1}^{n}$ and $f_{n}$ are contractions and hence continuous. We note that $\left\{\mathscr{R}_{i}, \Pi_{i-1}^{i}\right\}$ is an inverse mapping system $\Sigma$. The hypotheses (1) and (2) of Theorem 1 correspond to (1) and (2) of Theorem 5. By Theorem 5 the image of the limit space $\mathscr{R}$ of the inverse mapping system $\sum$ is dense in $\mathscr{W}^{\infty}$. This is just a statement that corresponding to any function $w(X)$ which defines a point $w$ of $\mathscr{W}{ }^{\infty}$ and to any $\left\{\varepsilon_{i}\right\}$ there exists $r(X)$ which determines a function of $\mathscr{R}_{n}$ for each $n$ such that $|r(X)-w(X)|<\varepsilon_{i}$ when $X \in W_{i}$. In this way Theorem 1 can be regarded as a special case of Theorem 5 . 


\section{REFERENCES}

1. W. Kaplan, Approximation by entire functions, Mich. Math. J. 3 (1955-6), 43-52.

2. P. W. Ketchum, On the possible rate of growth of an analytic function, Trans. Amer. Math. Soc. 49 (1941), 211-228.

3. S. Lefschetz, Algebraic topology, Amer. Math. Soc. Colloquium Publications, vol. 27, New York, 1942.

4. G. Mittag-Leffler, Sur la représentation analytique des fonctions monogènes uniformes d'une variable indépendante, Acta. Math. 4 (1884), 1-79.

5. A. Sinclair, Generalization of Runge's theorem to approximation by analytic functions, Trans. Amer. Math. Soc. 72 (1952), 148-164.

6. W. J. Thron, The theory of functions of a complex variable, John Wiley and Sons, Inc., 1953.

7. J. L. Walsh, Interpolation and approximation by rational functions in the complex domain, Amer. Math. Soc. Colloquium Publications, vol. 20, New York, 1935.

SOUTHERN ILLINOIS UNIVERSITY

CARBONDALE, ILLINOIS 